\section{Temporal lobe epilepsy is a progressive disorder}

\author{
Neda Bernasconi and Boris C. Bernhardt
}

We read with interest the commentary by Kuzniecky et al. (Epilepsy: Is localization-related epilepsy a progressive disorder? Maybe... Nat. Rev. Neurol. 5, 356-357 (2009) $)^{1}$ on our recently published work on MRI-based analysis of cortical thickness in patients with pharmacoresistant temporal lobe epilepsy (TLE). ${ }^{2}$ Using longitudinal and crosssectional designs, we showed that TLE is associated with progressive neocortical atrophy that is distinct from normal aging.

Kuzniecky et al. expressed concerns about the differences between normal aging-related cerebral atrophy observed in our healthy controls and those reported in a previous study, which showed widespread thinning across the entire cortex. ${ }^{3}$ In response, we would like to highlight a number of factors that could have contributed to the seemingly more restricted pattern of atrophy observed in our control group, and dispel the impression that effects of aging on cortical thickness were found only in patients and not in healthy controls.

One explanation that could account for the differences in cerebral atrophy observed between our study and previous studies might arise from the sample characteristics. Our control group had a mean age of 33 years (range 20-66 years), whereas other studies investigated cohorts with higher mean ages (mid 40s and higher), ${ }^{3,4}$ as well as more-extended age ranges. ${ }^{3,5}$ Thus, previous analyses might have observed more cortical thinning that was directly related to aging in later decades of life.

Another source of variation between our study and previous studies might arise from the choice of statistical methods employed in the analysis. We thresholded significant clusters using random field theory, ${ }^{6}$ which controls the chance of ever reporting a false positive result to $P<0.05$ despite large numbers of tests being performed. On the other hand, previous studies applied the false discovery rate (FDR) procedure that controls the proportion of false positives reported to be within $0.05 ., 4,7$ In the previous study highlighted by Kuzniecky et al. FDR-corrected maps showed moreextended regions of age-related cortical thinning across multiple cohorts when compared with findings at uncorrected thresholds of $P<0.001 .^{3}$ In our study, we presented cortical maps showing trends (uncorrected $P<0.005$ ) for negative effects of age on cortical thickness in controls. ${ }^{2}$ These areas corresponded, to a large extent, to those consistently reported in previous studies, including the superior temporal and prefrontal cortices. ${ }^{3}$ Moreover, an additional interaction analysis was designed to statistically highlight differences in aging effects between TLE patients and healthy controls. ${ }^{2}$ This analysis showed that cortical thinning in patients with TLE was considerably greater than cortical thinning associated with normal aging across extended neocortical regions.

The causal relationship between seizure activity and brain damage still needs to be clarified; however, our results together with previous findings demonstrating the association of cumulative structural and metabolic changes with TLE, ${ }^{8-11}$ provide compelling evidence that TLE is likely to be a progressive disorder.
Neuroimaging of Epilepsy Laboratory, Department of Neurology and McConnell Brain Imaging Center, Montreal Neurological Institute and Hospital, McGill University, 3801 University Street, H3A 2B4 Montreal, Quebec, Canada (N. Bernasconi, B. C. Bernhardt)

Correspondence to: N. Bernasconi neda@bic.mni.mcgill.ca

doi:10.1038/nrneurol.2009.82-c1

Competing interests

The authors declare no competing interests.

1. Kuzniecky, R., Thesen, T. \& Devinsky, O. Epilepsy: Is localization-related epilepsy a progressive disorder? Maybe... Nat. Rev. Neurol. 5, 356-357 (2009).

2. Bernhardt, B. C. et al. Longitudinal and crosssectional analysis of atrophy in pharmacoresistant temporal lobe epilepsy. Neurology 72, 1747-1754 (2009).

3. Fjell, A. M. et al. High consistency of regional cortical thinning in aging across multiple samples. Cereb. Cortex 19, 2001-2012 (2009).

4. Salat, D. H. et al. Thinning of the cerebral cortex in aging. Cereb. Cortex 14, 721-730 (2004).

5. Sowell, E. R. et al. Mapping cortical change across the human life span. Nat. Neurosci. 6 309-315 (2003).

6. Worsley, K. J., Andermann, M., Koulis, T., MacDonald, D. \& Evans, A. C. Detecting changes in nonisotropic images. Hum. Brain Mapp. 8, 98-101 (1999).

7. Benjamini, Y. \& Hochberg, Y. Controlling the false discovery rate: a practical and powerful approach to multiple testing. J. Royal Stat. Soc. 57, 289-300 (1995).

8. Briellmann, R. S., Berkovic, S. F., Syngeniotis, A., King, M. A. \& Jackson, G. D. Seizure-associated hippocampal volume loss: a longitudinal magnetic resonance study of temporal lobe epilepsy. Ann. Neurol. 51, 641-644 (2002).

9. Theodore, W. H. \& Gaillard, W. D. Association between hippocampal volume and epilepsy duration. Ann. Neurol. 46, 800 (1999).

10. Coan, A. C., Appenzeller, S., Bonilha, L., Li, L. M. \& Cendes, F. Seizure frequency and lateralization affect progression of atrophy in temporal lobe epilepsy. Neurology 73, 834-842 (2009).

11. Tasch, E. et al. Neuroimaging evidence of progressive neuronal loss and dysfunction in temporal lobe epilepsy. Ann. Neurol. 45, 568-576 (1999). 